\title{
Graphite floatation on a magma ocean and the fate of carbon during core formation
}

\section{H. Keppler ${ }^{1 *}$, G. Golabek ${ }^{1}$}

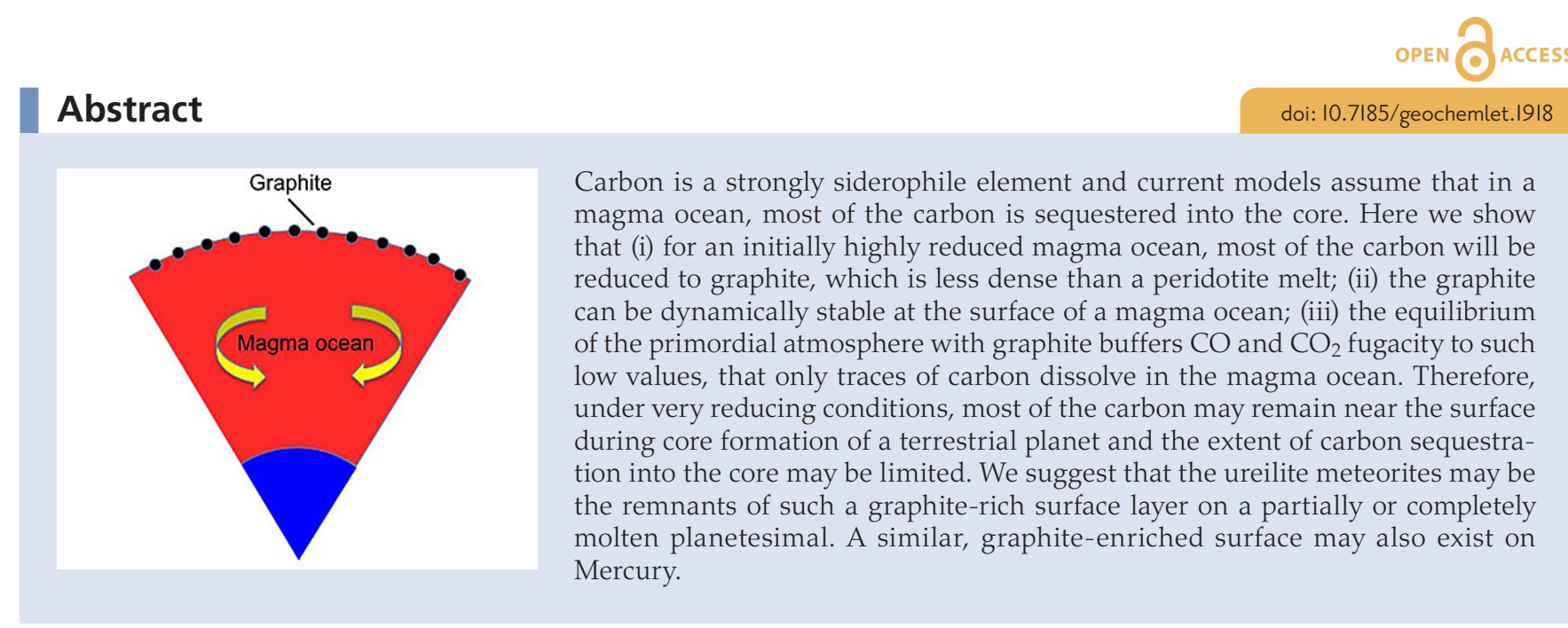

Received 21 March 2019 | Accepted 7 June 2019 | Published 9 July 2019

\section{Introduction}

The initial distribution of carbon in terrestrial planets was likely affected by processes occurring during the formation and solidification of a magma ocean (Elkins-Tanton, 2012). Carbon is known to be siderophile and a number of studies have therefore investigated the partitioning of carbon between a silicate melt and iron metal (Dasgupta and Walker 2008; Dasgupta et al., 2013; Chi et al., 2014). Measured partition coefficients $D_{C}{ }^{\text {metal/silicate }}$ typically range from 500 to 5000 . Accordingly, current models assume that during the evolution and solidification of the magma ocean, most of the carbon is sequestered into the core, leaving a carbon-depleted silicate mantle and primordial atmosphere behind (Dasgupta, 2013; Hirschmann, 2016).

The redox state of a magma ocean depends largely on the type of material accreted. Heterogeneous accretion models are required to meet constraints imposed by trace element concentrations in the present day mantle and experimentally determined trace element partition coefficients. The models of Rubie et al. (2011, 2015) suggest that Earth's formation started with the accretion of highly reduced material, while more oxidised material was added later. This corresponds to an initial oxygen fugacity $5.5 \mathrm{log}$ units below the iron-wustite buffer (IW-5.5), while during the late stages of accretion, oxygen fugacity increased to IW-2. During the later stages of accretion, most mass was delivered by large impactors and full equilibrium between metal and silicate melt was not achieved. Therefore, the partitioning of carbon into the core mostly occurred early in a very reducing environment. Carbon in chondritic meteorites is mostly present in the form of some carbonaceous, graphite-like material. Figure 1 illustrates the consequences of chemical equilibrium between a reduced magma ocean and graphite when a gas phase is present. The equilibrium between a $\mathrm{CO}-\mathrm{CO}_{2}$ gas phase and graphite is given by the CCO buffer. The oxygen fugacity imposed by this buffer depends strongly on the $\mathrm{CO}+\mathrm{CO}_{2}$ gas pressure. In equilibrium, the oxygen fugacity of the magma ocean, which is controlled by the concentration and redox state of iron, has to be the same as the oxygen fugacity of the CCO buffer. Figure 1 shows that for example at $2000 \mathrm{~K}$, a magma ocean with an oxygen fugacity $4 \mathrm{log}$ units below the $\mathrm{Fe}-\mathrm{FeO}$ (iron-wustite, IW) buffer would limit the $\mathrm{CO}+\mathrm{CO}_{2}$ pressure coexisting with graphite to 100 bars; at $1600 \mathrm{~K}$, the corresponding value would be just 10 bars. The carbon content in carbonaceous chondrites ranges from 0.1 to $>5$ wt. \%, with an average near 2.9 wt. \% (Kerridge, 1985). If the bulk Earth included just $2 \%$ of such volatile-rich material (Marty, 2012) and if all this carbon were converted into a $\mathrm{CO}-\mathrm{CO}_{2}$ gas phase, the resulting gas pressure would be in the range of 1600 to 2500 bar, depending on the $\mathrm{CO}_{2} / \mathrm{CO}$ ratio. Obviously, if oxygen fugacity is controlled by a reduced magma ocean, the resulting low $\mathrm{CO}+\mathrm{CO}_{2}$ pressures imply that most of the carbon must be reduced to graphite.

1. Bayerisches Geoinstitut, Universität Bayreuth, 95440 Bayreuth, Germany
* Corresponding author (email: hans.keppler@uni-bayreuth.de) 


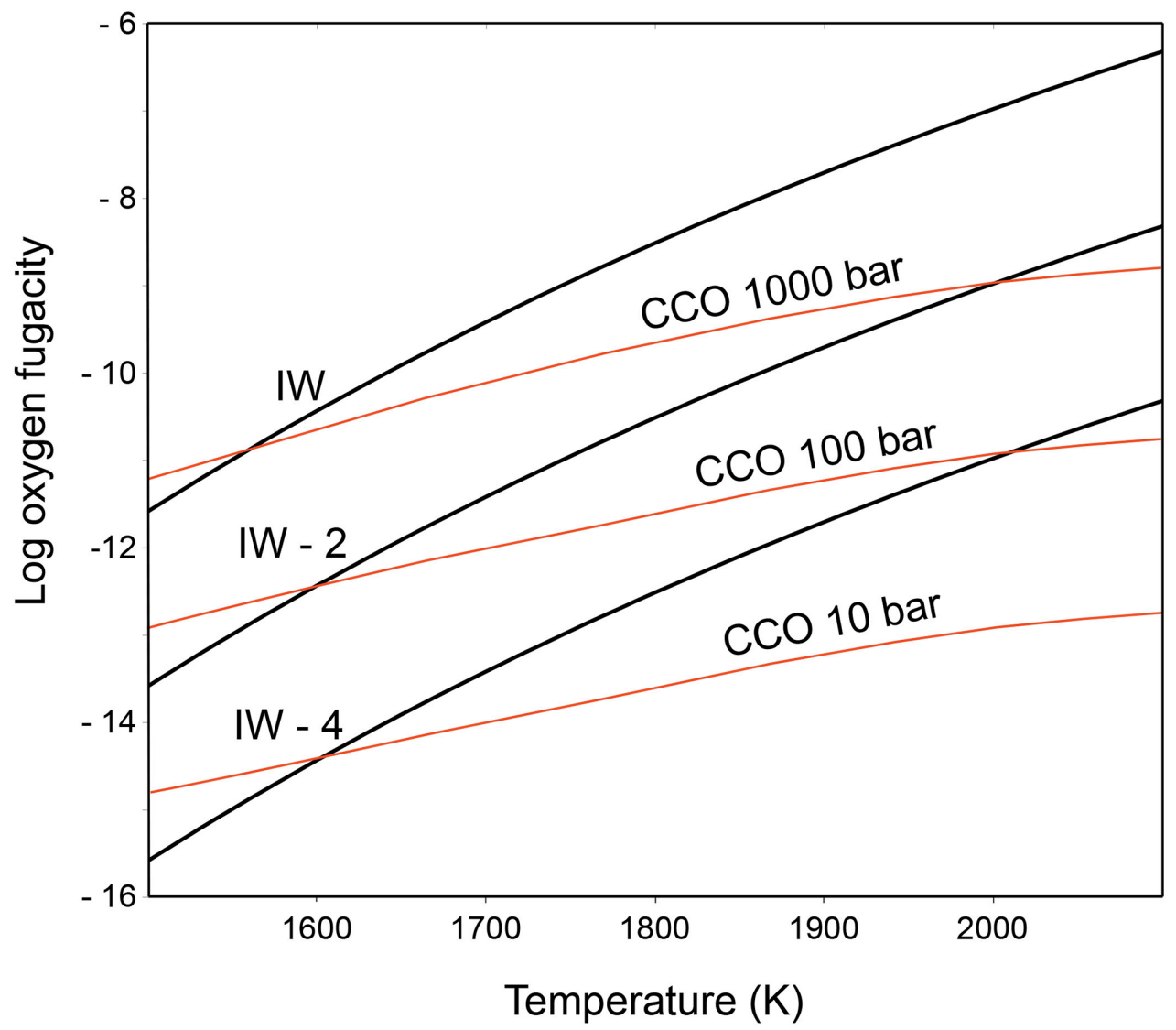

Figure 1 Consequences of thermodynamic equilibrium between a magma ocean, graphite, and a primordial atmosphere. Shown are the curves for the oxygen fugacities of the iron-wustite (Fe-FeO) buffer (IW) and for the graphite-CO-CO ${ }_{2}$ buffer (CCO) for different $\mathrm{CO}+\mathrm{CO}_{2}$ pressures (see Methods in Supplementary Information). Plausible oxygen fugacities of a magma ocean range from IW-2 to IW-5.5 (Rubie et al., 2011). The diagram shows that equilibrium between such a reducing magma ocean and graphite buffers the CO $+\mathrm{CO}_{2}$ pressure in a primordial atmosphere to very low values.

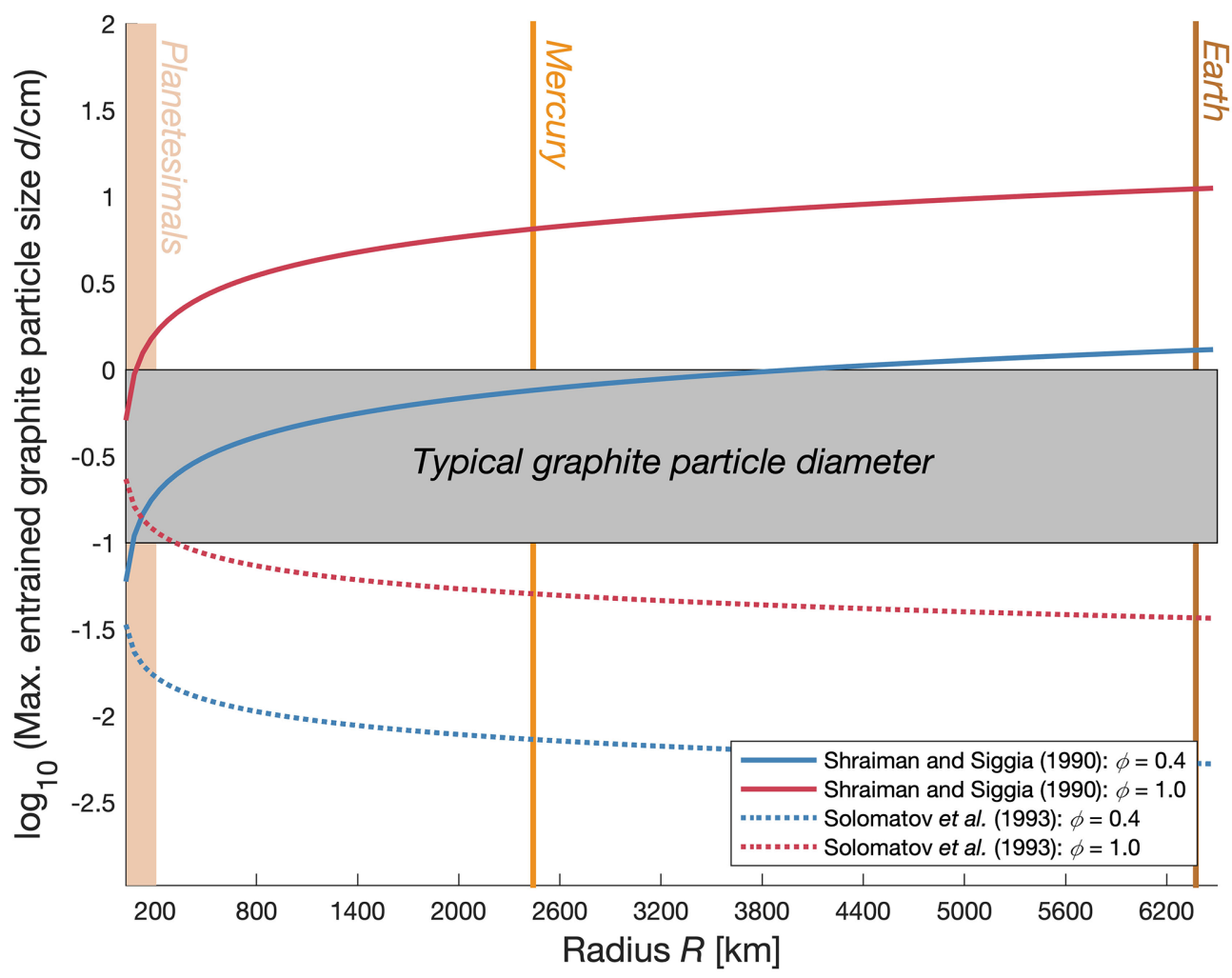

Figure 2 Two estimates for the diameter of the largest graphite particles that can be entrained in both a partially $(\varphi=0.4)$ and a fully molten $(\varphi=1.0)$ convecting magma ocean for planetary objects ranging from $25 \mathrm{~km}$ radius up to Earth's radius. The grey band indicates typical diameters of graphite particles found in meteorites. 


\section{Dynamic Stability of Graphite at the Surface of a Magma Ocean}

The density of graphite $\left(2250 \mathrm{~kg} / \mathrm{m}^{3}\right.$ at ambient conditions) is significantly smaller than that of peridotite melt $\left(2700 \mathrm{~kg} / \mathrm{m}^{3}\right.$ at $2000 \mathrm{~K}$; Courtial et al., 1997). Therefore, it appears plausible that graphite would float on the surface of a magma ocean. Indeed, the density difference between graphite and peridotite melt is much higher than that for plagioclase (with a density of $2600-2700 \mathrm{~kg} / \mathrm{m}^{3}$ at ambient conditions). Plagioclase floatation on a lunar magma ocean is widely believed to be responsible for the formation of the anorthosite crust of the Moon (e.g., Elkins-Tanton et al., 2011). Accordingly, it would be rather surprising if graphite did not accumulate at the surface of a magma ocean.

We have studied the potential rise of graphite particles in a magma ocean following the two approaches described by Solomatov et al. (1993) and by Shraiman and Siggia (1990); see Methods in Supplementary Information for details. For planetary objects ranging from a radius of $25 \mathrm{~km}$ up to that of the Earth, we estimated the maximum diameter of particles that can be entrained in the magma ocean and compare the results with the typical diameter of graphite grains or aggregates in meteorite samples (see Fig. 2). The results indicate that graphite particle floatation is viable when considering the equation given by Solomatov et al. (1993), while using the equation of Shraiman and Siggia (1990) graphite floatation would be only possible in small planetary objects. However, allowing for some sintering of graphite into larger aggregates at the surface of a magma ocean, graphite floatation will be possible in all cases.

\section{The Fate of Carbon in a Magma Ocean Covered by Floating Graphite}

Figure $3 a$ shows the fugacities of $\mathrm{CO}$ and $\mathrm{CO}_{2}$ in equilibrium with graphite at various oxygen fugacities and the resulting carbon solubility in the silicate melt (see Methods, Supplementary Information). Under very reducing conditions, predicted carbon solubilities are very low. At $2000 \mathrm{~K}$ and IW-6, only 0.15 ppm $C$ is in the melt. The solubility rises to about $1 \mathrm{ppm}$ near IW-4 and to $19 \mathrm{ppm}$ at IW-2. However, the partition coefficient of carbon between the metal phase and the silicate melt also depends on oxygen fugacity. Figure $3 b$ shows the predicted $D^{\text {metal/silicate }}$ according to the numerical regression model by Chi et al. (2014). For a peridotite melt with a structural parameter (non-bridging oxygen atoms per tetrahedron) of $\mathrm{NBO} / \mathrm{T}=3$, $2000 \mathrm{~K}$ and $3 \mathrm{GPa}$, the partition coefficient increases from about 1100 at IW-2 to $2.6 \times 10^{5}$ at IW-6. This means that the metal in equilibrium with the silicate melt should contain 2 4 wt. \% carbon, nearly independent of oxygen fugacity (Fig. $3 b)$. This shows that the thermodynamic models involved in the calculation are reliable. Since the metal phase is in equilibrium with the silicate melt, which in turn is in equilibrium with graphite, the carbon content in the molten metal should be the same as if the metal were in direct equilibrium with graphite. Indeed, the stable phase diagram of the $\mathrm{Fe}-\mathrm{C}$ system at ambient pressure shows that liquid iron in equilibrium with graphite contains between 5 and 6 wt. \% C at $2000 \mathrm{~K}$ (Okamoto et al., 1992). This is in satisfactory agreement with the predicted carbon content as shown in Figure 3b, considering that this calculation involves a rather large extrapolation of the partitioning model of Chi et al. (2014) to more reducing conditions.
The concentrations of carbon in metal as predicted in in Figure $3 b$ would imply that irrespective of redox conditions, most of the carbon in a growing planet would be sequestered into the core, if full thermodynamic equilibrium between the metal melt, the magma ocean and the atmosphere is reached, in agreement with previous models (Dasgupta, 2013; Hirschmann, 2016). Since the carbon concentration in the silicate melt in equilibrium with graphite is very low, however, this would require continuous replenishment of carbon in the melt during the entire time required to reach equilibrium with the metal. This is extremely unlikely to occur, since metal droplets in a magma ocean rapidly sink towards the core, while re-equilibration of the melt with the graphite and the atmosphere on the surface is only possible with a full convective overturn. Only if metal remained suspended in the melt during several convective overturns, could the predicted equilibrium concentration of carbon in the metal phase be reached. Due to the large density difference between metal and silicate melt, this is unlikely to occur, except perhaps for a small fraction or for very small droplets (see also Fig. 3 in Lichtenberg et al., 2018).

A more plausible scenario for the sequestration of carbon into the core is therefore that the metal phase during its descent towards the core only extracts the carbon that is already present in the silicate melt. In such a case, the possible concentrations of carbon in the metal are limited by mass balance. If for example, the mass ratio between newly added impactor metal and the mass of the magma ocean is 1:1000, and the melt contains $1 \mathrm{ppm}$ of carbon (at IW-4), then the maximum possible concentration of carbon in the metal is $1000 \mathrm{ppm}$, if all carbon completely partitioned into the metal. It should be noted that in this model, the possible concentration of carbon in the core is limited by incomplete equilibration with the graphite and the atmosphere on the surface; this is a completely different effect to the limited equilibration between metal and silicate melt (due to limited diffusion paths in either phase), which has been invoked in previous models (e.g., Rubie et al., 2011).

The maximum carbon concentration that may be reached in the core of a growing planet, if the accreted metal only extracts the carbon present in the silicate melt phase of the magma ocean, can be easily calculated. We assume that the accretion of a planet with final mass $M$ involves $N$ equalmass impact events. The metal added rapidly sinks to the core; after reaching the core, there is no further equilibration, as assumed in all current models of core formation (e.g., Rubie et al., 2011, 2015). The average carbon concentration in the core after complete accretion is then

$$
\overline{C_{m}}=c_{s}(N+1) \frac{1-f}{f}
$$

where $f$ is the fraction of metal in the impactor, which we assume to be constant and $c_{s}$ is the equilibrium concentration of carbon in the silicate melt (see Methods in the Supplementary Information for the derivation of this equation).

Figure 4 shows the predicted maximum carbon concentrations in the core for different oxygen fugacities as a function of the total number of impact events $N$. Accretion simulations (Rubie et al., 2015) suggest that for terrestrial planets, the number of impacts by planetary embryos ranges between 70 and 210, which is within the range covered by Figure 4. The metal fraction in the accreting material was assumed to be $f=0.32$, as in the Earth. Figure 4 also shows the fraction of total carbon in a planet ending up in the core, assuming a bulk carbon content of 500 ppm by weight, as inferred for the Earth (Marty, 2012). 

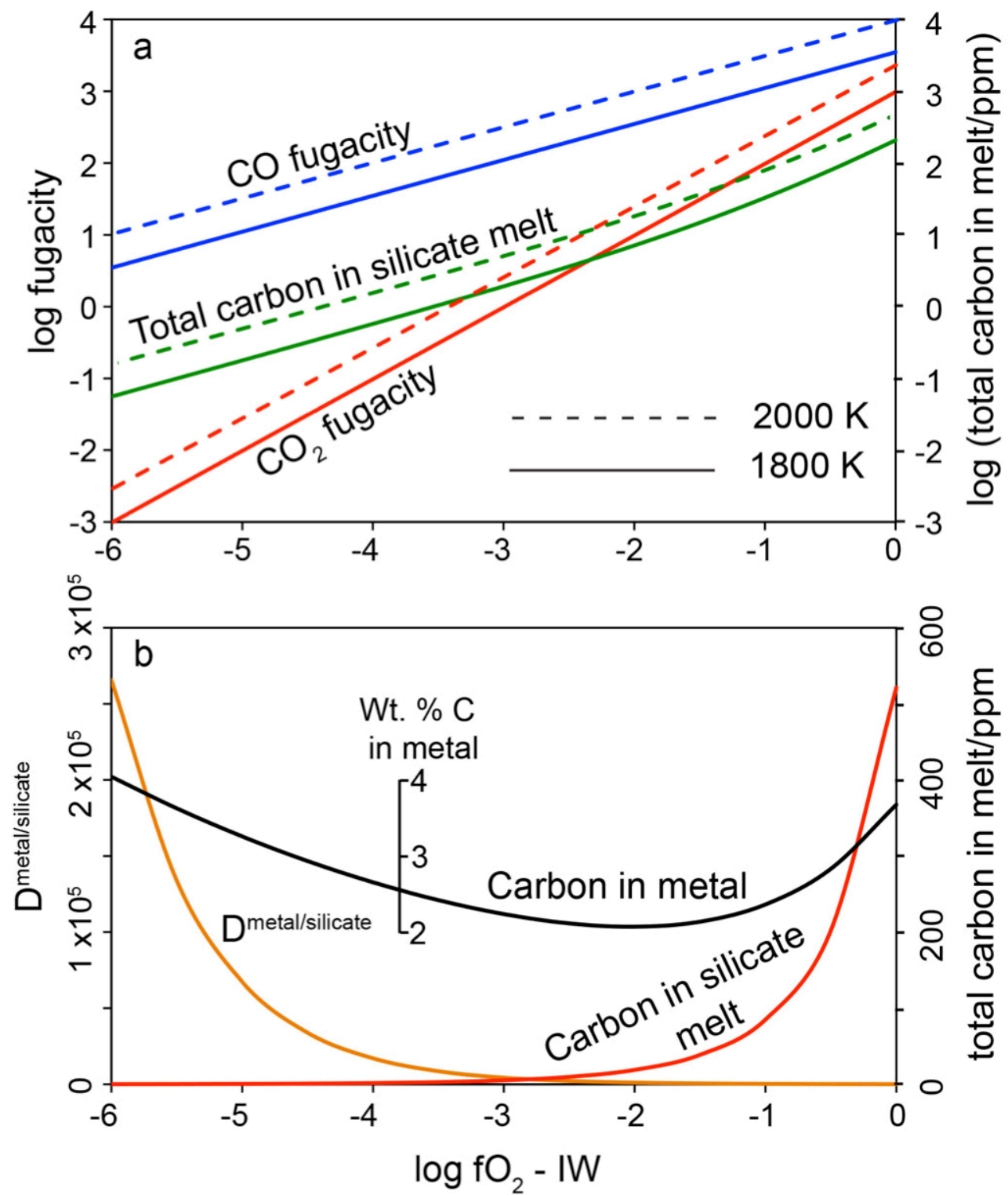

Figure 3 Partitioning of carbon between atmosphere, silicate melt, and metal in the presence of graphite. (a) $\mathrm{CO}_{\text {fugacity, }} \mathrm{CO}_{2}$ fugacity, and total carbon in a silicate melt in equilibrium with graphite for a temperature of 1800 and $2000 \mathrm{~K}$ at the surface of a magma ocean. (b) Carbon content in the silicate melt as expected from the equilibrium with graphite at $2000 \mathrm{~K}$ at the surface of a magma ocean, predicted metal/silicate melt partition coefficient of carbon inside the magma ocean (after Chi et al., 2014, NBO/T =3, $2000 \mathrm{~K}$ and $3 \mathrm{GPa}$ ) and predicted carbon content of metal in equilibrium with the silicate melt. In the presence of hydrogen, methane could be an additional carbon species; however, as we show in the Supplementary Information, the effect of $\mathrm{CH}_{4}$ on the behaviour of carbon is likely negligible.

\section{Carbon in the Early Earth, on Mercury, and a Possible Origin for Ureilites}

Figure 4 illustrates that the fate of carbon in a terrestrial planet strongly depends on the redox state prevailing during accretion and on the number of impacts of planetary embryos. Every impact sequestrates carbon from the graphite saturated magma ocean, thus the larger the number of individual impacts, the more efficiently is carbon transferred into the core. However, Figure 4 shows that even a very large number of impacts will only transfer $\approx 25 \%$ or less of the total carbon of a terrestrial planet into the core, if the oxidation state of the magma ocean is below IW-4. For IW-3, between about 25 and $75 \%$ of the total carbon may be sequestered into the core for a range of impact events between about 70 and 210 (Rubie et al., 2015). Only at a higher oxidation state of IW-2, could sequestration of carbon into the core be nearly complete. However, it is important to note that the curves given in Figure 4 are effectively upper limits of carbon concentrations in the core, as they assume that (i) the impacting metal extracts all carbon from the entire magma ocean and (ii) the metal fully equilibrates with the silicate melt. However, Deguen et al. (2011, 2014) show that for smaller impacts, the metal equilibrates only with part of the magma ocean and for large impacts only a fraction of the metal equilibrates. Both effects will limit the sequestration of carbon into the core, such that even at IW-2, some carbon will likely remain near the planetary surface.

For the Earth, accretion likely started at IW-5.5 with a gradual increase of oxidation state up to IW-2 during the final stage (Rubie et al., 2011). However, towards the end of accretion, above IW-3, equilibration of large impactor cores with the magma ocean became inefficient. Figure 4 therefore suggests that a significant fraction of total carbon, possibly as much as $50 \%$ did not enter the core. Since carbon solubility in the silicate melt is very low, this carbon would likely have remained as a graphite layer in equilibrium with a primordial atmosphere 


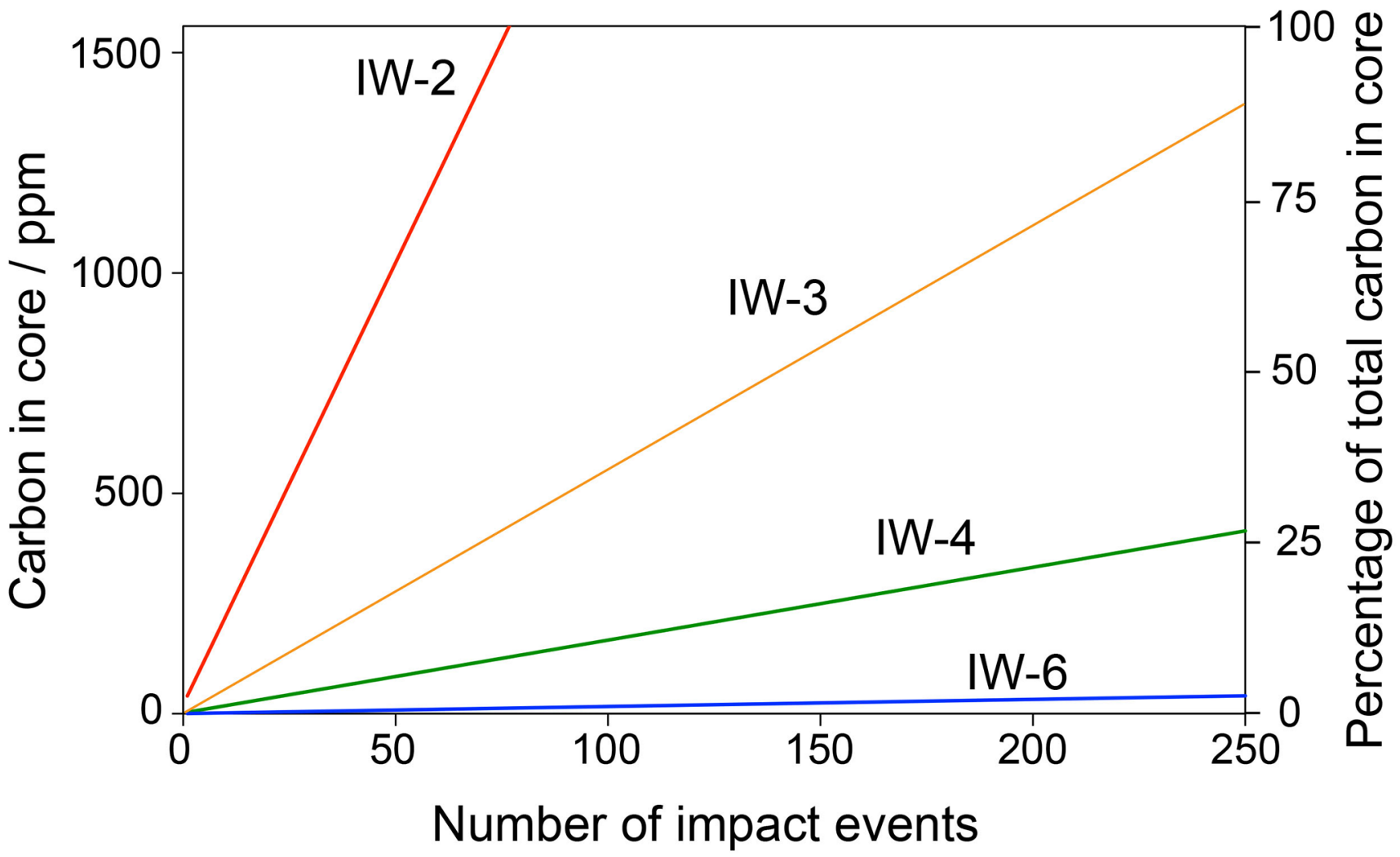

Figure 4 The fate of carbon during core formation of a terrestrial planet. Maximum possible carbon content in the core as a function of the number of impact events by planetary embryos, for oxygen fugacities from IW- 6 to IW- 2 and a surface temperature of $2000 \mathrm{~K}$ (left scale); predicted maximum fraction of total carbon sequestered in the core (right scale) for a terrestrial planet with a bulk carbon content of 500 ppm (Marty, 2012).

near the planetary surface. We suggest that the ureilites, a group of ultramafic, achondritic meteorites (Goodrich, 1992) that typically contain several percent of graphite and experienced at least some partial melting (e.g., Singletary and Grove, 2003; Wilson et al., 2008) may be the remnants of such a graphite-rich layer at the surface of a planet that went through a magma ocean stage involving graphite floatation on the surface. The carbon-rich nature of these meteorites may well be explained by the floatation of graphite at the surface of a magma ocean, where the graphite then got incorporated into an incompletely molten crust. Remote sensing data suggest that a similar carbon-enriched crust may exist on the surface of Mercury (Peplowski et al., 2016; see also Fig. 2 and the Supplementary Information).

\section{Conclusions}

We show that the equilibrium between a highly reducing magma ocean and a primordial, carbon-rich atmosphere necessarily leads to the precipitation of graphite, floating on top of the magma ocean. The graphite buffers the solubility of carbon in the silicate melt to such low values that the sequestration of carbon into the core becomes inefficient. A terrestrial planet may therefore solidify with a graphite-rich layer at the surface that retains most of the carbon inventory. This layer may reduce planetary albedo and therefore enhance surface temperatures. Moreover, the concentration of graphite near the surface may be a viable mechanism for the selective loss of carbon by impact erosion. The ureilite meteorites may be samples of the graphite-enriched surface of an asteroid that experienced a magma ocean stage.

\section{Acknowledgements}

Constructive reviews by Bruno Scaillet and an anonymous referee helped to improve this manuscript.

Editor: Ambre Luguet

\section{Additional Information}

Supplementary Information accompanies this letter at http:// www.geochemicalperspectivesletters.org/article1918.

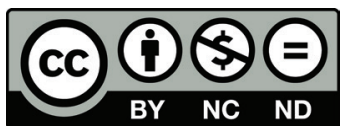

This work is distributed under the Creative Commons Attribution Non-Commercial No-Derivatives 4.0 License, which permits unrestricted distribution provided the original author and source are credited. The material may not be adapted (remixed, transformed or built upon) or used for commercial purposes without written permission from the author. Additional information is available at http://www.geochemicalperspectivesletters.org/ copyright-and-permissions.

Cite this letter as: Keppler, H., Golabek, G. (2019) Graphite floatation on a magma ocean and the fate of carbon during core formation. Geochem. Persp. Let. 11, 12-17.

\section{References}

Chi, H., Dasgupta, R., Duncan, M.S., Shimizu, N. (2014) Partitioning of carbon between Fe-rich alloy melt and silicate melt in a magma ocean - Implications for the abundance and origin of volatiles in Earth, Mars, and the Moon. Geochimica et Cosmochimica Acta 139, 447-471. 
Courtial, P., Ohtani, E. Dingwell, D.B. (1997) High-temperature densities of some mantle melts. Geochimica et Cosmochimica Acta 61, 3111-3119.

DASGUPTA, R. (2013) Ingassing, storage, and outgassing of terrestrial carbon through geologic time. Reviews in Mineralogy and Geochemistry 75, 183-229.

DASGUPTA, R., WALKER, D. (2008) Carbon solubility in core melts in a shallow magma ocean environment and distribution of carbon between the Earth's core and the mantle. Geochimica et Cosmochimica Acta 72, 4627-4641.

Dasgupta, R., Chi, H., Shimizu, N., BuOno, A.S., Walker, D. (2013) Carbon solution and partitioning between metallic and silicate melts in a shallow magma ocean: Implications for the origin and distribution of terrestrial carbon. Geochimica et Cosmochimica Acta 102, 191-212.

Deguen, R., Olson, P., Cardin, P. (2011) Experiments on turbulent metal-silicate mixing in a magma ocean. Earth and Planetary Science Letters 310, 303-313.

Deguen, R., Landeau, M., Olson, P. (2014) Turbulent metal-silicate mixing, fragmentation, and equilibration in magma oceans. Earth and Planetary Science Letters 391, 274-228.

ELKINS-TANTON, L.T. (2012) Magma oceans in the inner solar system. Annual Review of Earth and Planetary Sciences 40, 113-139.

Elkins-Tanton, L.T., Burgess, S., YIN, Q.Z. (2011) The lunar magma ocean: Reconciling the solidification process with lunar petrology and geochronology. Earth and Planetary Science Letters 304, 326-336.

GoOdRICH, C.A. (1992) Ureilites - a critical-review. Meteoritics 27, 327-352.

HiRsCHMANN, M.M. (2016) Constraints on the early delivery and fractionation of Earth's major volatiles from $\mathrm{C} / \mathrm{H}, \mathrm{C} / \mathrm{N}$, and $\mathrm{C} / \mathrm{S}$ ratios. American Mineralogist 101, 540-553.

KerRIDGE, J.F. (1985) Carbon, hydrogen and nitrogen in carbonaceous chondrites: Abundances and isotopic compositions in bulk samples. Geochimica et Cosmochimica Acta 49, 1707-1714.

LichtenberG, T. Golabek, G.J., Dullemond, C.P., Schönbächler, M. GERYA, T.V., MEYER, M.R. (2018) Impact splash chondrule formation during planetesimal recycling. Icarus 302, 27-43.

MARTY, B. (2012) The origins and concentrations of water, carbon, nitrogen and noble gases on Earth. Earth and Planetary Science Letters 313-314, 56-66.

Oкамото, H. (1992) The C-Fe (carbon-iron) system. Journal of Phase Equilibria 13, 543-565.

Peplowski, P.N., Klima, R.L., Lawrence, D.J., Ernst, C.M., Denevi, B.W., Frank, E.A., Goldsten, J.O., Murchie, S.L., NitTler, L.R., SOLOMON, S.C. (2016) Remote sensing evidence for an ancient carbon-bearing crust on Mercury. Nature Geoscience 9, 273-276.

Rubie, D.C., Frost, D.J., Mann, U., Asahara, Y., Nimmo, F., Tsuno, K., Kegler, P., Holzheid, A., Palme, H. (2011) Heterogeneous accretion, composition and core-mantle differentiation of the Earth. Earth and Planetary Science Letters 301, 31-42.

Rubie, D.C., Jacobson, S.A., Morbidelli, A., O’Brien, D.P., Young, E.D., Devries, J., Nimmo, F., Palme, H., Frost, D.J. (2015) Accretion and differentiation of the terrestrial planets with implications for the compositions of early-formed Solar System bodies and accretion of water. Icarus 248, 89-108.

SHRAIMAN, B.I., SigGIA, E.D. (1990) Heat transport in high-Rayleigh-number convection. Physical Review A 42, 3650-3653.

SingLETARY, S.J., GROVE, T.L. (2003) Early petrologic processes on the ureilite parent body. Meteoritics and Planetary Science, 38, 95-108.

Solomatov, V.S., Olson, P., Stevenson, D.J. (1993) Entrainment from a bed of particles by thermal convection. Earth and Planetary Science Letters 120, 387-393.

Wilson, L., GOODRICH, C.A., VAN ORMAN, J.A. (2008) Thermal evolution and physics of melt extraction on the ureilite parent body. Geochimica et Cosmochimica Acta 72, 6154-6176. 